mediastudies.press • Social Media \& the Self: An Open Reader

\title{
“Do I Look Like My Selfie?”: Filters and the Digital- Forensic Gaze
}

Christine Lavrence ${ }^{1}$, Carolina Cambre ${ }^{2}$

${ }^{1}$ King's University College at Western University, ${ }^{2}$ Concordia University

Published on: Oct 24, 2020

License: Creative Commons Attribution-NonCommercial 4.0 International License (CC-BYNC 4.0). 


\begin{abstract}
Filtered faces are some of the most heavily engaged photos on social media. The vast majority of literature on selfies have focused on self-reported practices of creating and posting selfies and how subjects view themselves, but research on using filters and the kinds of looking filter provoke is underexplored. Part of a larger project, this analysis draws from a study using photo-elicitation techniques to discuss selfie filters with 12 focus groups, exploring the dominant discourses of cis-gendered looking within digital sociality. We explore how participants edit their selfies, imagine potential audiences, interact with, and perceive the filtering behaviors of others, asking what the "work" of filters is, visually and socially. We probe the kinds of discourses filters participate in, and their gendered and affective dimensions. Our focus groups indicate that when looking at the selfies of others there is often an a priori assumption that filtering has been applied, whether conspicuously or not, to the extent that visual tune-ups have become central to the genre itself. As such, we explore the ambivalence and anxiety about authenticity that filters produce, as well as the intense looking practices aimed at decoding the legitimacy of images. We posit that filters are part of a digital ecosystem that demands an intensification of looking practices, which produce and enhance specific forms of objectification directed toward selves and others within digital environments.
\end{abstract}

Keywords: selfies, digital sociality, filters, Instagram

All of life is based on semblance, art, deception, points of view [Optik i.e. perspective], and the necessity of perspectives and error. (F. Nietzsche Birth of Tragedy [Nietzsche, 2000, p. 7])

It's super ironic because I posted a selfie on Instagram . . . and the caption was like "baby keep it simple" but the funny thing was that I edited the shit out of it (group laughter) . . . it was the most "not simple," not at all . . . I edited it for two hours plus some. (Research participant)

\title{
Introduction $\underline{1}$
}

THE ABILITY TO manipulate one's appearance with selfie filters is intuitively appealing and lends itself to identity work, play, and self-exploration. In the vast majority of the literature on selfies, studies have focused on self-reported practices of creating and posting selfies and how subjects view themselves, but research on using 
filters and the kinds of looking consequently provoked is underexplored. $\underline{\text { Charlotte }}$ Leclercq_(2016). who examines the emergence of selfie filters on Snapchat calls for further research within this area and stresses the lack of critical insight into the roles of filters in social media practices. Given that images of filtered faces are some of the most heavily engaged photos on social media, and that they are central to digital selfpresentation, the dearth of research is surprising and something we hope to begin to address here. Part of a larger project, this analysis draws from a study using photoelicitation techniques to discuss filters with 12 focus groups of four to five participants. Here, we discuss the project's second phase and the dominant discourses of cisgendered looking within digital sociality. $\underline{2}$ In this context, we explore how participants edit their selfies, imagine potential audiences, interact with, and perceive the filtering behaviors of others by asking what the "work" of filters is, visually and socially. We wonder about the kinds of discourses filters participate in, their gendered and affective dimensions and we examine filters beyond surface appearances of how one person might "see" another.

Not only is filter usage ubiquitous within selfie practices, but we also learned that when looking at the selfies of others there is an a priori assumption that filtering has been applied, whether conspicuously or not, to the extent that visual tune-ups have become central to the genre itself. Selfie filters allow for the production of serial "versions" of the self, generating the ongoing engagement necessary for establishing and maintaining visibility and "relevance" within social media ecologies. While the introduction of selfie filters initially produced conspicuous, playful forms of editing animated by the novelty of the apps, (i.e., dog face filter), a shift to subtler, ambient forms of editing, where it is not always obvious, has become normalized and entrenched within social media ecologies. As such, looking at selfies invites us to attend to degrees and types of filtering at work in the pictures, and to negotiate their implicit authenticity claims, where participants are repeatedly asking: What do you really look like?

In this article, we reflect on filter use and the aesthetic and affective labor of selfies, production processes, ambivalence and anxiety about authenticity, and the intense looking practices aimed at decoding the legitimacy of images. We posit that filters are part of a digital ecosystem $\underline{3}$ that demands an intensification of looking practices, which produce and enhance specific forms of objectification directed toward selves and others within digital environments. Animating this looking is the intent to decipher the authenticity $\underline{4}$ and artifice at play in the image, which pulses throughout the transcripts 
from our empirical research and is expressed through taken for granted "rules" about what is considered acceptable when filtering the selfie. Our questions then become: What kinds of regimes of looking might be enacted through filters? How do filters complicate the notions of authenticity within social media ideologies, where participation and visibility are understood as tools of personal and spontaneous selfexpression?

We position selfies relationally and processually as quasi-immediate networked social practices enacted between people within mediating ecologies involving online/offline intersections and tensions. Selfies are a distinctive genre from traditional selfportraiture produced through various media (Cambre \& Lavrence, 2019), which we now operationalize as inclusive of the plandid $\underline{5}$ because our participants discussed plandids as selfies. That is, they did not specifically differentiate between images that were plandids and those that were selfies. The "plandid" - a portmanteau of the words "planned" and "candid" (Diamond,2017)—existed prior to the digital shifts producing "selfie" and in current image-fluent digital contexts has increased in popularity. No longer simply a planned candid, plandids are carefully choreographed photos of a person posing in what is often referred to by participants as a mini-photo-shoot, usually without acknowledging the camera, while looking and/or doing something fabulous. The plandid has been called the "super-selfie" (Diamond, 2017), since the photo taken by another person (or using a timer) allows for significant staging and the enactment of a variety of scenes. It is highly stylized, borrows iconographic conventions from advertising and fashion, and is considered more sophisticated than a selfie. Individuals no longer hold the phone, allowing for a "natural" aesthetic that ideally "should look as though you weren't aware you were getting your photo taken" (heng, 2017). This move de-emphasizes faces, permits more background content, and thematic consumption (e.g., travel, pleasure, luxury, and lifestyle) in addition to bodily capital. Arguably, plandids require more resources, time, and attention to detail than traditional selfies. Reflecting this, the advice literature suggests using "high speed sequential shooting" to take the "hundreds of photos" required to get the "perfect plandid shot." 6 Our focus group participants often discussed selfies and plandids interchangeably. Yet they still felt incentivized to shoot selfies, which are the most heavily engaged images on Instagram and receive the most likes and comments. The availability of these easy likes was modulated by the implicit "rules" of Instagram dictating that selfies be posted sparingly_too many, especially when filters are conspicuously applied, are considered gauche and "thirsty" for attention. 


\section{Filters, Faces, Power}

Photographic filtration is not isolated to digital image-making; lens filtration and earlier forms of photo manipulation can be traced throughout the history of photography. In 1974, Bryce Bayer invented the filter that allowed photographs to be colored, followed by the invention of the first black and white digital camera in 1975 by Steven Sasson and Gareth Lloyd (ㅂevesi,_2012). In 1987, the Knoll brothers released Photoshop, which was sold to Adobe in 1988 (Britannica, 2019) and then Photoshop's features became widely available as they were implemented in mobile apps that were accessible and affordable for amateur users. Later, iPhone released a software development kit (SDK) in 2008, which commercialized and popularized photographic filtration. Since then, accessible photo editing applications have become central to selfie production (e.g., Snapchat, Instagram, Facetune, Facetune2, VSCO, AirBrush, YouCam, and others). Many filters involve the use of facial recognition software that identifies a face in the camera and "adds a real-time virtual layer on top" with "real time feedback on the screen [that] enables users to playfully interact with these filters and effects" (ㄷhipper (2018), p. 5). The editing apps range in their affordances from the color filters and presets of VSCO and Lightroom, to the visual effects, stickers and geofilters of Snapchat, and to facial recognition apps such as Facetune and $\mathrm{AI}$, and facial editing apps like FaceApp that allow for more comprehensive and structural edits where the face's features and proportions can be more fully reconfigured.

Research participants used the terms filtering and editing interchangeably, rarely differentiating between them. While recognizing this slippage, we follow suit in our analysis for two reasons: first, ready-made filters (e.g., Coachella) were more likely to be referred to as masks and second because even editing that seems manual because a user is making numerous decisions, is still pre-cooked to the extent that color palettes and sliding scale options match visual codes of what has been already deemed by software designers as "correcting" images. In either case, a form of looking at the image of self and other is activated that requires specific theorization. Based on these transcripts, we acknowledge filters as pivotal in the iconographic conventions of selfies: They allow for the seriality and variability of self-presentation that social media requires to achieve and maintain online visibility. Filters facilitate playful virtual "dress up" while also producing coded self-presentations. Filters also help manage the vulnerability that visibility produces-for example, women describe sending men filtered selfies (i.e., dog face) when they don't have makeup on. Filters have an affinity with neoliberal subjectivities, where everyone is seen as able (and obliged) to achieve 
"beauty" through vigilant bodywork, so that the failure to do so is relentlessly critiqued (see Evans \& Riley, 2013).

While research suggests photos involving faces are $38 \%$ more likely to get likes and $32 \%$ more likely to get comments (Bakhshi et al.,2014, p. 965), filtered photos are $21 \%$ more likely to be viewed and $45 \%$ more likely to be commented on (see Bakhshi et al. (2015), p. 1). Bakhshi et al. (2015). found that photographs with warm tones, high exposure, and high contrasts are especially effective in increasing engagement and describe filters as a form of digital "crafting" that make photos "more special or cherished" and "self-made" (p. 2). Conversely, we wonder about the depersonalizing and homogenizing effects of filters. For example, skin smoothing editing apps render faces slightly blurry, so that the shape and size of facial features are difficult to discern, rendering faces more generic looking, or "same-ing" them. For Barnard,_2016, selfies produce the "[dis]empowerment paradox" where filters can be as-if liberating while simultaneously heteronormative, and signaling a disconnect between "material and affective conceptions of empowerment" (p. 63).

Moreover, many filters automatically lighten skin, thin bodies, narrow noses, remove wrinkles, and more. Algorithmic actions mark filters as racialized, gendered, and normative: They tell us about who is welcomed to participate and considered worthy of digital visibility. It bears reiterating that this phenomenon is raced, gendered, classed, aged, abled. Filters monitor and keep out unwelcome visual content and implicit forms of personhood. As Rettberg_(2014). notes, filters underscore how "the image is not entirely ours" (p. 26). Along this vein, Elias and Gill (2018). identify ways filters encode heteronormative and racialized disciplinary practices by lightening skin, reinforcing idealized "waist-to-hip ratios," and the "aesthetic surveillance and labour" that postfeminism demands of its subjects (pp. 67 and 68). Their claim that editing apps signal an "unprecedented regulatory gaze upon women" (Elias and Gill, p. 59) is supported by empirical evidence reporting that women use filters more than men (Dhir et al., $\underline{2016}$, p. 551).

\section{Theoretical Frame: Looking/Gender/Selfies}

We situate this study in relation to a growing body of critical literature exploring the tensions and contradictions within selfie practices (Barnard, 2016; Cambre \& Lavrence,2019; Kedzior \& Allen,2016; Vivienne,2017). We also engage literatures on post-feminist subjectivities (Gill’s (2007), 2016, 2017; Gill \& Scharff, 2011) to explore gendered practices of looking linked with "the construction of a visual cyber- 
subjectivity" (ingrose \& Coleman, 2013, p. 132). While many theorize women as objects of the male gaze, iley et al.,2016 stress that the "role of women's looking in subject formation" (p. 96) is under-researched yet crucial. These looking practices are "socially constituted, relational, judgmental, and regulatory: a powerful dialogic of looks that holds out the promise of recognition of successful femininity and the threat of failure" (Riley_et al.,2016, p. 99) They not only reiterate an older notion that appearance is the key basis of social value for women (Riley et al.,2016, p. 94) but also recast validation for appearance as a primary source of female empowerment through ideologies of online visibility.

Our study therefore resonates with the body of critically informed research that has emerged examining a "fascinating looking" at women within post-feminism (McRobbie, 2009, p. 100, cited in Riley et al.,2016, p. 98). We employ Gill's (2007). definition of post-feminism as a "sensibility" in order to understand the postfeminist shift where women's bodies have become their primary "source of power" (p. 149) recasting "sexual objectification" as the "freely chosen wish of active, confident, assertive female subjects" (p. 153). However, we also noted that while our female cis-gender participants were more attentive to the selfies coded as female, our male cis-gender participants mirrored this homosocial looking and were more attentive to the selfies coded as male. Patterns of looking enacted between cis men within our empirical research pathologized selfies and especially filtered selfies as "feminine" practices, provoking a regulatory gaze within male peer interactions and protecting masculinist self-presentation. As such, throughout the transcripts participants described intense and critical looking practices that were especially, but not exclusively, homosocial and collective looking rituals that were distinct from those of non-binary participants.

\section{The Gaze, the Forensic Eye, and the Digital-Forensic Gaze}

The focus group transcripts mapped out a set of visual, aesthetic, and social codes about the use of filters, which were described with surprising consistency across age, location, and gender. While Senft's (2015). phenomenological analysis points to the tactility of the mobile phone interface, as the user touches and manipulates the screen, the intensity of the scrutiny and investments that looking practices engendered in our participants suggest broader social relations. Given this, we return by necessity to the critical purchase of theorizing the "gaze" as it allows us to explore structures of desire, difference, and power that animate looking. A vast literature exists on the notion of the gaze across Western academic disciplines since the 1950s. We briefly touch on some relevant ideas and thematic currents in order to build some of our own ideas. Jean Paul 
Sartre (1943/1957). devoted a section of his philosophy of consciousness, Being and Nothingness to "The Look," where he relates the feeling of being caught in the gaze of another. He describes intently looking through a keyhole, realizing someone is in the room watching him, and then feeling embarrassed and objectified. For Sartre, the gaze is intimately knotted into tensions between objectification and subjecthood. Vividly illustrating this, his 1944 play No Exit features three characters that have died and are trapped in a room that is their hell. At one point Estelle says,

When I can't see myself I begin to wonder if I really and truly exist. I pat myself just to make sure, but it doesn't help much. I've six big mirrors in my bedroom . . . When I talked to people I always made sure there was one near by in which I could see myself. I watched myself talking. And somehow it kept me alert, seeing myself as the others saw me.

Paradoxically, only by objectifying herself through the as-if gaze of another could she allow herself a form of derivative subjecthood, which meant adopting values that others used to qualify her. Sartre's theory of the gaze has been under-estimated in what it can offer new directions in thinking of relationships between watching and objectification. Instead, the trajectory of Western ideas moves quickly to work like Jacques Lacan's (1978). iteration of the gaze in psychoanalytic theory, where anxiety becomes a central thematic. A limitation for Lacan, as for Sartre, writing within "the terms of a masculinist culture" (Judith Butler (1990), p. vii) is that desire is "presumed as heterosexual and masculine" (Judith Butler (1990), p. vii).

When Foucault (1973). writes "Panopticism," the link between power and the gaze is made explicit. He positions the gaze, and even the possibility of being subjected to a surveilling gaze as the medium of power to which individuals submit. Similarly, in describing the power dynamics between doctors and patients, Foucault (1973). used the medical gaze to conceptualize the knowledge/power equated with the diagnostic eye. Bringing together feminist sensibilities with aspects of Lacanian psychoanalytic theory and power in Foucault's work, film theorist Mulvey's (1989). develops the notion of "the male gaze" to explain how camerawork in cinema adopts a male subject position that views women as objects to be looked at, voiding their subjectivity, and that of female spectators in theaters who must also, thanks to the camera's eye, view women on screen through this gendered lens. By contrast, philosopher Judith Butler (1990). describes a feminine gaze that reverses and "inexplicably returns the glance, and contests the place and authority of the masculine position" (p. vii). 
Mulvey's (1989). controversial "male gaze" was also contested by bell hooks' notion of "the oppositional gaze" (hooks, 1992) where she reclaimed space for spectators to resist passive viewing. In other elaborations of the gaze, such as Ann Kaplan's (1997). imperial gaze, Edward Said's (1978). post-colonial gaze, the ideas of objectification, the male gaze, and power remain central, yet over time more attention is given to the racialized and Westernized regulatory and normalizing effects of looking. Because of the way the mechanisms of the gaze are positioned, as in through cameras, hidden eyes or simply the creation of the sensation of being seen rarely do those being viewed have the ability to "look back" at the viewer, underscoring asymmetrical power relations. Throughout these 20th-century theoretical configurations, the subject is consistently positioned as a unitary whole and thus falls short of what understanding 21st-century online looking practices now demand.

The analysis of online looking is also informed by the broader, historical relationships between photography and evidentiary practices. Theorizing the "forensic eye," Graybill, 2019 traces the evolution of 19th-century crime scene photography and the emergence of forensic identification techniques such as fingerprinting (p. 103), which signals a shift toward more positivist judicial practices (p. 97). Crime scene photography promised empirical evidence of crime using techniques like "perspectometric" framing to create an appearance of scientific neutrality (Graybill,_ 2019). Graybill traces the rhetorical underpinnings and effects of these techniques, which enabled a photo's viewers to "animate and imagine" (p. 114) the crime. Under the guise of providing clinical objectivity, crime scene photographs were intended for more than preserving evidence. Rather, they co-created an "arena of public conviction" (p. 96) by participating actively in the construction of "judicial meaning," serving as "engines of imagination and emotion, as much as of empirical fact" (p. 114). By coding and encourage specific ways of looking, these images were designed to render a crime "legible" (p. 105).

The 19th-century forensic gaze tethers photography to the search for transgression, where "the clinical precision of the image" allows viewers to "examine the body with the same cold attention we give the inanimate objects around her" (Graybill, 2019, p. 94). Similarly, photographic portraits served phrenological and "physiognomic" projects identifying criminality through facial features (ekula, 1986, p. 12). Sekula alerts readers here to the paradoxical relationship between the "ameliorative" and "repressive" aspects of photography, which were noted by its earliest advocates, who "struck up a Benthamite chorus, stressing the medium's promise for a social calculus of pleasure and discipline" (p. 8). 
The oscillation between adjudication and emotion, pleasure and discipline, restoration and repression, creating a both/and in practices of online looking are, we claim, animated by what we call a "digital-forensic gaze" that presumes the use of filters: as such, selfie looking practices are highly sensitized to the tensions between artifice and authenticity. While the terms suggest a dissective and clinical form of looking, the negotiation of truth and beauty is driven by and productive of anxiety, envy, desire, disgust, shame, and fear so that adjudication and feeling inform each other. In the context of cis-gender sociality, this gaze reflects the "exclusionary and injurious practices" of gendered "normative cruelties" (Ringrose \& Renold, 2010, p. 575). This gaze is highly astute, deconstructive, playful, deeply normative, and at times, cruel.

Etymologically, "forensic" from the Latin for "of the market place, public" stresses a "belonging to" public discussion and debate (Merriam-Webster). Similarly, "visual cyber-subjectivity" (Ringrose \& Coleman, 2013) is expressed through rituals of public assessments via like-counts and comments, but also signals the market ethos and the "ambient" (Jurgenson \& Rey,2010; Rey, 2012) production that saturates online looking. The filtered selfie is presumed to be both "real" and yet "not actual" (Deleuze

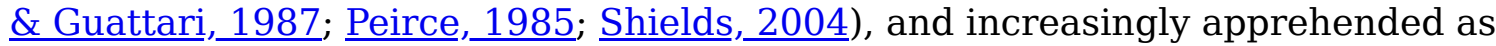
legitimate if it can be "filtered to look natural." We situate the digital-forensic gaze within this dialogic relation, where the viewer's gaze responds to digital images and "confirms that something has happened." As relational practices, selfies involve posting and sharing, seeking input and "the completion of meaning" from viewers-a face has presented as "pretty," a trip was "fun," a relationship is "happy." And while the viewer witnesses and confirms the photograph's veracity, the viewing subject experiences a schism, whereby the digital-forensic gaze is also paradoxically trained to incriminate and look for signs of pathology.

Our research indicates that when looking at selfies, the use of filters was usually presumed, and deciphering authenticity is integral to what drives looking practices. This habituated practice of scrutinizing selfies, the digital-forensic gaze, is at least partially animated by decoding the filtration effects applied (or not) in the image. We use digital-forensic not only to recall early photographic practices but also to link with Elias and Gill (2018). astute observations related to how the mobile camera lends itself to the "forensic scrutiny of the female body" (p. 60). Elias and Gill (2018). explore how "beauty apps not only recalibrate but also reconfigure the gendered rationality of postfeminist self-capitalization, predicated as it is on relentless beauty surveillance, labour and optimal transformation through consumption" (p. 60). We build on this by exploring the looking practices that selfie filters generate through qualitative research, 
broadening the analysis to include the looking directed at and between both women and men and examine how ideas of authenticity are negotiated. Looking at selfies is skeptical (i.e., highly sensitized to artifice, difference, power, desire, and transgression) and yet also credulous: Again there is a paradoxical fracturing of subjectivity. In other words, this digital-forensic gaze assumes the presence of artifice and yet somehow feels the image as if it were real, which creates affective ruptures or psychic pressure points that push their way into these discussions. The notion that photographs are evidentiary artifacts sits alongside the notion that they are aspirational and performative. Moreover, this looking is regulatory and pleasurable, serious and playful, dissective and affective at the same time, and highly social.

The digital-forensic gaze thus asks, "What is suspect in this image and what evidence of editing/filtering can be ascertained? How does the selfie give away its artifice and betray its "as-if" effortlessly natural curation?" Our study suggests that the most critiqued selfie transgression is conspicuous filtering: whereas subtle filtering that passes as "natural" is considered ideal. As such, authenticity is not positioned on a binary system that distinguishes between unfiltered selfies as natural and filtered selfies as "fake." Given this, the digital-forensic gaze is underpinned by both ontological questions asking whether what appears in the selfie is true or real, and aesthetic judgments asking whether what appears is good or beautiful. Filters entangle these questions, such that what is edited well (i.e., creates a natural aesthetic) and decoded as conventionally beautiful is often understood as true and good. Our research participants were skilled at decoding internal contradictions and evidence of editing in pictures (sometimes called "watermarking?")-blurry facial features as a result of skin smoothing, the warped lines on walls in the background when bodies have been thinned. While conspicuous editing delegitimize selfies, this is not that case for selfies edited to appear "natural": as one of our participants said, "you want the selfie to be so real even though it might be edited [. . .] that's the goalto edit it so well it looks real."

\section{Methodology}

For over 2 years we have conducted focus group interviews in three Canadian cities with people between the ages of 18 and 30, totaling 23 focus groups containing four to five participants each across three project phases. We began our research with focus groups that collected stories of selfie-taking, which led to our interest in filters. In this analysis, we focus on our second phase of research where we conducted 12 focus groups using photo-elicitation methods, and showed research participants six publicly 
available selfies, and discussed what they saw. The pictures shown represented diverse faces along the lines of race, and gender, while ability questions were invisible and the age range was similar to that of participants. Five images had different filters applied, while the sixth had no filter applied. Consistently, focus groups addressed the images in the first 15-20 min and then spring-boarded into more general discussions around platform behaviors and expectations as well as personal storytelling. We trained four research assistants similar in age and gender identities as participant groups, and then we conducted a focus group with three of the facilitators themselves to debrief their experiences, which was especially productive. Focus groups consisted of racially diverse cis-gender women, cis-gender men, and individuals who identified as nonbinary or alternatively abled. (For a detailed discussion see $\underline{\text { Cambre \& Lavrence, 2018.) }}$

\section{Data Collection and Analysis}

Using photo-elicitation, "a process of organizing interviews around photographs" (Harper 2002, p. 16), to explore subjectivities produced and enacted through filters allowed open-ended image-prompted discussion with minimal direction. Through images, notions and meaning systems that may already be present for participants (Becker,1974; Pauwels, 2011) were provoked. As visual sociologist Luc Pauwels (2015). observes, "visual materials [are] used as 'stimuli'” that for participants "may include pre-existing 'societal imagery' [. . .] as well as researcher or respondentgenerated materials" (p. 67). Because these photos are public and easily found online using reverse image searches, we exclude the images from this publication to protect the identities of the individuals they portray. At the same time as preserving openness to indigenous concepts, our theoretical framework is sensitized to aspects of personhood in the neoliberal, post-feminist, affective, and material ecology that renders possible and normalizes the use of selfie filters.

Throughout our process, we attend to moments of "affective intensities" (Ringrose \& $\underline{\text { Renold }}, \underline{2014}$ ), as well as gaps in discussions to plan our next step of data collection

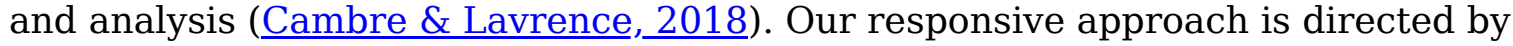
an encounter with the problem, a deliberately slow qualitative research practice done "without cutting corners where corners matter" (Bal, 2002, p. 3). In other words, we delve into the transcripts and recordings without pre-cooked content coding categories, and without a reductive approach to analysis simplistically relying only on word repetition. Instead, we engage a post-structural genealogical practice of marking mentions of people, objects, affects/relations, and events/happenings to position each researcher's "gaze as focalizer" (Bal,_2002, p. 38) attending to continuities, 
discontinuities, and ruptures, noting that these markers can overlap. Each researcher traveled through transcripts individually first, then we met, compared, discussed, and interrogated our interpretive processes. We located word repetition as a signal of an intensity, or a pulse to listen to, rather than assuming an equivalency with a theme.

In our discussion below, we explore three central problematics: (1) questions of authenticity that filtered selfies generate, (2) the discourse of "trying too hard" in selfies, and (3) the gendered patterns of self-presentation and looking within which these questions of authenticity and "trying too hard" are situated. Our analysis attends to how looking behaviors between people were reported, and within the conversations, the qualifications, the pauses, the equivocations, the jokes, as well as how word-affect relationships create meanings for participants. We emphasize the sociality of looking directed at selfies and understand focus group methodologies as crucial for examining "shared and tacit beliefs" (Gilbert, 2017, p. 343) as manifested through interactions with others. We also trace shared discourses activated through conversations that meandered, told stories, paused, and provoked emotions.

As noted, while participants initially responded to the six images, broader discussion was generated about how participants themselves use and experience filters, much of which was prescriptive in nature. A normative framework of filtering became apparent, further illustrating how visual images as methodological tools operate as distancing techniques to manifest personal narratives and insider discourses. By using qualitative research to work with images and words, we traced the multiple visions of participants activated via different perspectives. In asking what responses the selfie pictures triggered in our participants, we aimed to defamiliarize conventional ways of seeing (and not seeing) (see Natali, 2019).

\section{Discussion and Analysis}

\section{Authenticity/Gender/Trying Too Hard}

The two most frequently mentioned platforms in our study were Snapchat and Instagram, which were consistently described as requiring different filtering practices. Snapchat was described as space for playful, silly pictures shared between friends, whereas the stakes of Instagram were understood to be high, requiring strategy and calculation. At the core of Instagram's algorithmic imaginary is an unquestioned belief in the value of numerical growth and visibility. Reflecting this, Instagram was described as the frontline of self-presentation, where friends, acquaintances, 
strangers, potential love interests, and employers might make quick assessments, and was reserved for the "best" and most curated pictures, akin to a highlights show reel. Snapchat's "fun" and "cute" filters (i.e., dog faces, flower crowns, eyeglass stickers) were considered inappropriate for Instagram. Evolving social norms of platform behaviors require that filtering is subtle filter to signal that "you know what you're doing" (P2). As one participant stated, the goal of Instagram is "to make yourself look more natural, that's what you can do with editing" (P2). These ontological ambiguities are epitomized in the distinction between "Rinsta" and "Finsta" Instagram accounts. Rinsta (combining "real" with "Insta/gram") is a public account reserved for the most curated images: "Finsta" (combining "fake" and "Instagram") is a private account with fewer followers, typically close friends, allowing for more relational and less visual and scripted practices. Finsta accounts also permit a broader range of affect, where users can share more freely, without the pressures of looking good and growing followers, while shielded from parents or unwanted viewers. A blogger writing about Instagram has a conversation with a teen who comments:

My real Instagram account is kind of fake-like, only pictures of my "best" life . . . I use my Finsta to share funny, inside jokes with my closest friends. My real Instagram has like, 400 followers. My fake one only has like, eight. (․ㅏㅇㅣ, 2019)

Somehow, within this ecology, the "real" Instagram account is where the pictures are "fake"-suggesting that the images may be staged but "real" in their effects. Research participants shared fluency in the visual codes of filters, most of them easily identified which filters were being used in the photos shown to them, the platforms they belonged to and the conventions of their usage. The transcripts indicated that although there is a great deal of playful social interaction enacted through filtered selfies, however there is also the equally heavy risk of stigma when using them, which we will explore in what follows. For participants, celebrities and influencers were seen as being given permission to post selfies with conspicuous filtering because of their status and social capital, resonating with Bridges and Pascoe's (2014). insight that the flexibility of identity is more readily available to those with privilege. Research participants often avoided speaking directly about the extent to which they themselves used filters in their own selfies, answering with qualifications, contradictions, distancing strategies, and hesitations. For example, someone might claim at they do not use filters, yet further on in the transcript relate how they use them.

These frequent contradictions signal ontological ambiguities and we ask what actually counts as edited or filtered in these discussions? Conversely, we wonder what 
"natural" is taken to mean, given it was used within and across focus groups to describe the ideal filtered selfie? If edits are inconspicuous or natural looking, do the pictures count as a filter/edited? This is not self-evident in these discussions, and closer analysis indicated that our participants did not conceptualize filter usage along a binary where the image is either digitally altered or not. Rather within the Instagram ecology, filtering practices are positioned on a continuum ranging from "natural looking" to conspicuous and "fake"-unfiltered selfies are outliers and their status as unfiltered is never fully known. These ambiguities are reflected in a conversation about using filters for "retouching" or making minor "corrections":

Joelle ${ }^{8}$ : Just like little blemishes -

Meghan: Exactly!

Joelle: Just like little things that don't really change how you look, they're just changing one little thing that was off in that moment

Meghan: Exactly like, I use um, airbrush like, if I have pimples, I'll cover them up, or like I use the detail thing for my lashes if I want them to look more like extra, but I don't change anything, like I don't add anything onto it, you know what I mean?

Joelle: No I do the same, it's completely fair!

In this discussion, participants stressed a distinction between "changing" how you look and "correcting" something that was "off in the moment" or wanting to look a "little extra." Corrections are described as "fair," or not cheating, which signals the competitive nature of these practices and the implicit rules governing them. However, where and how "corrections" become "changes" in the editing process is unclear.

As "staged performance[s]" selfies articulate, "a destabilized sense of self, one that is caught between competing ideals and stylizations" (ㅂess, 2015, pp. 1632, 1635). This is enhanced by the often multiple layers of filtering, as one participant reflects:

sometimes like I'll take a photo on Snapchat that will have my face filter, and then I'll add a filter on top of that and then I'll take a screenshot of that and put it on Instagram and then put on an Instagram filter on that.

The "self" represented in the photo is clearly understood as carefully crafted and aestheticized, and presenting visually in a particular moment (i.e., in this particular 
light, from this angle, with this arrangement of facial features, body parts) as afforded through a particular technological arrangement (i.e., with this light filters, editing apps, or camera). Digital photography allows for this careful parsing out of instances and potentialities, producing many possible variations of the self's image and the availability of the face for scrutiny: manifested through time and expertise, emphasizing the ephemeral, and lived experience as much as it does calculation. Moreover, the radically intimate gesture of photographing the face at close range, which as Schipper (2018). established, is accentuated when using filters, raises the stakes of personal authenticity.

Reflecting these tensions, something akin to a golden rule is inscribed throughout these discussions: "Your selfie should never make it look like you're trying too hard. It should look like you snapped that photo casually" (Peterson, n.d.). "Trying too hard" was how selfies were discredited, manifesting within our transcripts with comments like: “[she’s] trying too hard, which I personally don't like”; “it's like [he's] clearly trying to get someone's attention." "Trying too hard" was coded as appearing "fake," "insecure," and behind the trends like "duckface" and/or using too many hashtags. Filters played a central theme in these discussions about "trying too hard," specifically, when they were used too often and too conspicuously. Many participants distanced themselves from these behaviors, positioning them as appropriate for young teens, social media novices, reflecting the expectation that selfie behaviors would become more sophisticated and subtle as people entered their 20 s.

An exchange between participants in reference to a selfie of a young woman using the Coachella crown Snapchat filter states,

Alyssa: Aren't we over the super filtered snapchat flower crown thing? Is this a post on Instagram because seriously it's so tacky and overdone, like I think that she's probably pretty but I can't say for sure because of how much editing is going on with that filter (group of participants agree).

Olivia: Yeah but like anyone can use a filter, it's just that it's overdone and so fake that it's not even like reality, like why post that when everyone knows you're so edited and probably don't look like that. Everyone looks pretty with it trust me, I know some not very cute girls that use it and look so pretty but then you see them and it's like oh, it's just the filter!

Later another participant says, 
Lauren: She is trying so hard but she looks like she's actually really pretty but you don't really know because everyone (spoken very dramatically) looks good with the filter.

Here, participants cannot fully determine exactly the extent of edits and whether or not the woman in the selfie is in fact "pretty." The participants question why she would post this picture when "everyone knows" it is filtered, and its appearance as "pretty" is delegitimized by the judgment that "everyone looks pretty" when using that filter. This idea echoed throughout the transcripts: "that Snapchat filter makes everyone look pretty, when we all look like thumbs," "Everybody looks pretty with that filter, so I really don't know what you look like" or "who looks bad with that on?" The ability to signal "distinction" (Bourdieu, 1984), understood here as effortless beauty is eroded with the sense that "anyone" can achieve it with the right curation effects.

Shared discursive terms and values indicated that patterns of looking were encoded in highly gendered ways, especially in the intense scrutiny enacted between women within each female-centered focus group. Those discussions were animated by attempts to decipher signs of artifice, cheating and whether someone is "really" pretty or if it is just the filter. The judging gaze that dissects the image and its accompanying discourse was taken as given, as legitimate by all focus group members, that is, in no instance was anyone taken to task for cutting into the subjects of the pictures, or criticizing specific practices the digital-forensic gaze was a given.

In other words, social media ideologies that equate visibility and the "control" women purportedly "exert over their bodies" with empowerment, and the recreational peer surveillance that these platforms normalizes, results in the "obfuscation" and inability to critique the male gaze (Winch, 2012, p. n/a) and looking practices more broadly. Yet the term that female participants playfully describe their own looking as "creeping" suggests that a sense there is something unsavory or asymmetrical about looking. A female participant states, "I feel like girls creep each other more than guys." Later on in the focus group they reiterate the notion that creeping is gendered behavior stating,

Jessica: Girls stalk so much better man! (group laughing).

Samantha: NSA, FBI hire us! (group laughing).

In another focus group, a female participant brings out her phone to show the others an Instagram feed of a mutual acquaintance, $\underline{9}$ gazing intensely at the screen the conversation: 
Alyssa: Isn't she perfect? She's legit my girl crush

Olivia: She's like perfect [. . .]

Lauren: Are her boobs real? [. . .] Does she look the same in person?

Alyssa: Yeah, she's so pretty [. . .] I live for this shit, like creeping is the best.

Lauren: All I do on Insta is creep other girls.

Natasha and Alyssa: (speaking together): Same.

Alyssa: That's what Instagram is for.

We noted the emotional intensity-eruptions of laughter and excited conversationthese conversations provoked. The admiration the participants shows a particular Instagram feed is followed by a digital-forensic check-“are her boobs real?": "does she look the same in person?" While participants were reluctant to admit the extent of their own filtering, they were surprisingly open about the pleasures of looking. The term creeping suggests a slow, "fascinated" mode of looking. There is a sense that a great deal of time, emotion, and thought is invested in looking at both friends and strangers.

Another conversation in a different focus group that references the Instagram account of a mutual acquaintance is relevant here:

Samantha: Filters changes your face shape, it changes your eyes, your lips, everything [. . .] you push and pull things in different directions [. . .] every photo I see I think "oh my god you're so pretty" and then I see them in person and I feel more normal now. Like "you're on my level, you're not this goddess that I see on Instagram." (group agrees and says "yeah" in background)

Beatrice: This one girl that's notorious for that, you know who I'm talking about?

Samantha: Just say it!

Beatrice: Okay (she names a woman on Instagram).

Alexis: (gasps) I was just creeping her before I came here, I shit you not!

Participants: (excitedly talking over each other) 
Samantha: Yeah she's beautiful and then in person she doesn't even look like herself at all (dramatic tone).

Shared values around creeping (in the sense of lurking, or stalking) inform the use of the term and are an integral part of peer dynamics here. The conversation makes evident the deep investments these participants have in their looking-they erupt into excited conversation simultaneously as they discuss a particular acquaintance they "creep." Again, we see the digital-forensic comparison between online and offline appearance, and as they assess the feed's authenticity, we glimpse the anxiety linked with these images with phrases like "you're on my level, not this goddess I see on Instagram." When participants describe the incongruence between someone's online and offline appearance, the conclusion is that "she's beautiful and then in person she doesn't even look like herself at all." Their judgment signals the anxiety and shame that they too might be assessed as inauthentic and as such "attention-starved" and "too much" (see Senft, 2018). Notably, the person offline does not look like the edited face in the photo that has become the reference point for "herself," prompting the question-“does she look like her selfie?” Grogan et al. (2018). argue that female selfie takers understand differences between "their "unreal", digitally manipulated, online selfies" and their "real" offline identities, and use filters to present normatively attractive self-images (p.15). Nonetheless, we wonder whether it matters that they understand the difference if they experience the picture as-if it were real. In this sense, as they detect the failure of their mutual acquaintance to live up her online pictures, we see that although the digital-forensic gaze is skeptical about what it sees, on another level, it takes what it sees at face-value and feels the image as if it were real. Illustrative of this, a female participant says,

We're pulling back from the whole fake thing and we're calling each other out and we're being more real [. . .] she wants to post her thing, and if this is how she looks without a filter, that's great. But everybody is just so wary of everybody right now, that we're like "Oh she has a filter, she's fake” but we're still looking at her [thinking] "she's so pretty." (Amanda)

This participant describes the shift to less conspicuous filtering, the lack of trust and the culture of "calling each other out" on social media. She is savvy, acknowledging that while skeptical, her eye registers the "fake" picture as unattainably "pretty." As such, the knowing self-betrayal, or trust/not-trust rupture presents a similar internal contradiction. Just like a plandid is a contradiction, and not just a portmanteau, a picture literally cannot be both planned and at the same time candid. What happens in 
that paradoxical rupture? The oxymoron has been collapsed right into the word in a way that it is somehow masked, it is self-cancelling in its very essence and accepted uncomfortably at first, until its paradoxes become invisible through use.

The digital-forensic gaze is also enacted by men and directed both toward other men and women. Filters were understood heteronormatively in these discussions, described as digital makeup, such that cis-gender men described feeling prohibited from using filters, unless they did so ironically ensuring that they are not taking the selfie "too seriously." One participant describes his experiences:

Ethan: When I was younger, number one, taking selfies would have been a very feminine act. Number two, to use a filter would have been unspeakably feminine for a man . . e emasculating.

Similarly, another participant describes his use of filters being policed by peers:

Gabriel: I remember I was just like taking one of those doggie filter selfies or whatever just for fun, and my friend was like "yo, what are you doing?" (disapproving tone), and I was like "I'm ... I'm just taking a dog picture" and he was just like "ugh, okay (disgusted tone) why are you doing that?" and I'm like "I don't know, it's just funny."

Describing the use of filters as "unspeakably feminine" and "emasculating" affirms the idea that cis-gender women use filters more than cis-gender men. When a friend questions his filter use, our participant's immediate response is to assure his friend that it is "just for fun" and "it's just funny" thus shoring up his masculinity by stressing he is not taking it seriously. Another participant describes being on the other end of this kind of exchange and says "I mean if one of my friends has the filter, I'd give him a little playful shot, like 'oh my god dude that dog filter? Sodumb'." This gender bias is encoded into the affordances of this filter which often assume the face is female and "feminizes" it by extending the eyelashes, widening and enlarging the eyes, and narrowing the chin. Cis-gender women in our study also conveyed the sense that they did not understand men as using or needing filters, rather the opposite: something was wrong if they did. As one participant commented,

Maria: a guy snapchatted me with a filter and I messaged my friend like "ew, a guy messaged me with a filter," like I hated it, I don't think a guy should look like a little kitty cat [. . . ] I think for a straight guy it's safer just to not use the filter, 
but why are the filters geared towards females though? Because they know that we want to look perfect and guys don't care?

While she questions why the filters are "geared towards females," she is also actively disgusted when a male uses one. This is not the only time in our transcripts where male heterosexuality is questioned because of filter use. The above comment also affirms the extent to which online looking is peer-based, people look and decode together: she texts her friends to recount a guy friend's filtering transgression. Our participants often pulled out their phones and scrolled through feeds in order to show examples and illustrate particular points. They described soliciting help from friends when editing or looking for selfie captions, messaging friends about their Instagram posts for private feedback, or collective rituals scrutinizing selfie editing.

The transcripts indicate that filters are pathologized in ways associated with feminine stereotypes-as signaling weakness, insecurity, and dishonesty. Our cis-male participants reacted negatively to the selfies of women that were conspicuously filtered and instead expressed preference for selfies that appeared "naturally" pretty. At stake, was wanting to know what the women "really" looked like so that filters were understood as a masking strategy. A conversation between our male participants is as follows:

Matthew: When I'd see a girl that has like, first picture's a filter picture, I'd swipe left, like "nah I'm good" (participants laugh).

David: Yeah like, what do they actually look like?

Matthew: I wanna see what you look like, are you gonna walk around with a flower crown on your head all day?

Interviewer: So it's kind of like an avoidant type thing?

Participants: (visible and audible agreement)

David: It's like a cute way for them to be like "I'm kinda ugly but I don't want you to see." (participants laugh)

The laughter among these participants stands in stark contrast to the anxiety and feelings of pressure expressed by female participants and then they continue:

Matthew: You're pretty because we can't see you (laughter) 
David: Everyone's pretty with a paper bag on their head, right?

The filter is likened to a "paper bag" over the head-the pressure to be continuously visible, to "look good" and appear "natural," and to render oneself vulnerable to the dissecting and consuming gaze of the other permeates this sociality. The cis-gender women in our focus groups were keenly aware that men react negatively to filters and judge them as a way to "hide" undesirable features or mask themselves at times when they are not "looking their best." For example, a participant states,

You know when you're snapchatting a guy and you're like oh shit, I don't wanna always use the filter because guys judge on that so much, so you're like, I have to put on a little bit of something (group laughs and agrees) to not look like a little gremlin. (Lauren)

The use of the filter can be read as providing a moment of refuge from the normative pressures of this visual economy. But is this reprieve akin to a moment of agency? Or does it underscore the relentless scrutiny of the digital gaze, if the refuge afforded is accompanied by a recasting of the face within heteronormatively attractive forms and if this masking reiterates and re-naturalizes (ironically) these normative pressures? It becomes difficult to distinguish between the disciplinary and the agential as the gendered power relations involved in the demand to "send me your picture" becomes normalized within "visibility as empowerment" social media ideology. This discourse says that confident women are always available and willing to render themselves visible. Our transcripts complicate the notion that this visibility is always freely chosen and instead, complex structures of constraint work alongside and with nuanced negotiations of the terms of visibility.

This sense of "control" is also double-edged in that it generates anxiety about the ability to measure up to our "better" online selves. As some of our female participants discussed,

Joelle: I was just talking to my friend about this the other day, like I feel like it's so scary like we were talking about, like, do I look like my Instagram? Because like

Maria: You wanna know and make sure!

Joelle: Exactly!

Maria: You wanna make sure you're not catfishing! 
Joelle: Exactly I don't wanna catfish and like that's like one of my biggest fears, which is why again I think that when people use snapchat filters they change your face so much, so, and when they post that on Instagram like it's just like it's not really what you look like.

Maria: It's like everybody looks pretty with them so I really don't know what you look like.

Joelle: Exactly! And that's scary, I feel like it's so scary to-for like other people to see me in real life and go like "oh my god you're not as pretty as I thought you were."

Intersections between presumed perceptions online and offline in these interviews were fraught with tension and anxiety. The extent to which the photograph is "a

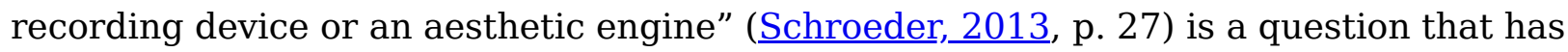
long troubled photography, and we see how this tension is at the core of selfie subjectivities. These anxieties prompt intensive visual practices of "checking" ("do I look like my Instagram?") that reinforce and amplify these tensions in a vicious circle of looking, feeling and (self) objectification.

\section{Conclusion}

Through this analysis we explored the rich discussions that were generated in our empirical research when we showed groups of people images of filtered selfies. We explored the aesthetic and affective labor of producing selfies as well as the centrality of filtering to these practices. These discussions indicate a fluid and evolving technosocial ecology where the introduction of selfie filters initially encouraged playful and conspicuous forms of editing, driven by the novelty they afforded but has shifted toward subtler, ambient forms of editing that have become deeply naturalized. As filtering shifted from "play" to allowing for the curation of a "natural aesthetic," a series of ontological ambiguities have emerged such that the distinctions between edited and non-edited, natural and a "natural aesthetic" within social media visuality ceases to be self-evident, requiring new forms of theorizing in relation to the tensions and ruptures this generates. The evidentiary nature of photos has become unsettled in the proliferation of edited images, producing intense looking practices and affective engagements issuing from the simultaneous credulity and skepticism of the eye, the identity work that selfies afford and the peer relations that constitute the context of looking. 
We developed the term, "digital-forensic gaze" to describe the condition and practices of looking that presume the images of people they see on social media are filtered, as well as the affective ecology that sustains this looking. These "digital-forensic" forms of looking and feeling intensify (self) objectification and train the eye to look more critically at the images of the self and others, asking what has been or might potentially be "fixed" or "edited": as one participant noted, "you take the filter off your face and you're like, now I just feel ugly." Within a context where neoliberal subjectivities position everyone as able and obligated to achieve "beauty" through vigilant bodywork (Evans \& Riley, 2013), selfie filters provide the tools for achieving and policing this work. Within this ecology, the face is less a marker of identity than a gesture toward a better, future version. Face-work on Instagram is therefore never done-as a participant comments: "Honestly, Insta is exhausting." While our participants were highly skilled at decoding artifice, as editing technologies become more sophisticated, it becomes increasingly difficult to accurately identify if and which filters have been used. Filters therefore generate individual and shared practices of negotiating, and assessing the legitimacy of digital self-presentation, a dissective gaze that we maintain enhances objectification, cultivates an ecology of heightened affectivity and is heavily gendered. While conspicuous filter use is heavily criticized across genders for being "fake" and "overdone," cis women are also continuously incentivised for using them, as their likes increase in proportion to their selfies' proximity to heteronormative beauty standards. Lines between "cheating" or "enhancing," "hiding" or just "looking a little extra" are shifting, contextual and relational in these transcripts. Our research suggests that using and engaging filters allows for play and "fun" but is also deeply anxiety provoking for cis women, as they worry about their ability to live up to their online selves, such that power and pleasure intermingle in the shape-shifting that filters afford, and in the fixing and undoing of meaning within selfie sociality.

\section{Author Contribution}

Authors contributed equally to this collaborative article. The use of the pronoun "we" in this article refers to the authors and not a disembodied universal "we."

\section{Declaration of Conflicting Interests}

The author(s) declared no potential conflicts of interest with respect to the research, authorship, and/or publication of this article. 


\section{Funding}

The author(s) received the following financial support for the research, authorship, and/ or publication of this article: Concordia University SEED fund (Internal); King's University College Research Grant (Internal); Fonds de recherche du Québec - Société et culture.

\section{Author Biographies}

Christine Lavrence is associate professor in the Department of Sociology at King's University College at Western University. Lavrence's research explores questions related to culture, identity, gender, visuality, consumption, memory, and memorialization from an interdisciplinary perspective.

Carolina Cambre is associate professor in the Department of Education at Concordia University. Cambre's research investigates the politics of communication, representation, critical visual sociology, and image theories. She looks at issues of representation using processual semiotics, anthropological and sociological theories through various critical frameworks.

\section{REPRINT}

"Do I Look Like My Selfie?': Filters and the Digital-Forensic Gaze" (Christine Lavrence and Carolina Cambre, Social Media + Society 6, no. 4, October 24, 2020)

OPENLY LICENSED

\section{Footnotes}

1. We are grateful to the anonymous reviewers for their insightful and productive suggestions. $\_$

2. Our work with participants who identify as non-binary, or who refuse to identify, indicates altogether different discourses and looking practices, which warrant being addressed with an entire article. As this leg of our research is still in a preliminary phase, this analysis is limited to cis gender men and women. $\_$

3. Granted the filters industry (cf. https://edition.cnn.com/2019/08/13/tech/snapchatlens-creators/index.html) and its prosumer developments are very influential, but fall outside the scope of this article, which focuses on user experiences. $\triangleq$ 
4. Authenticity is a contested term. For our purposes here, and based on the empirical work we are doing, we use it in Lindholm's (2013) sense: that when something or someone is authentic it suggests, "They are what they purport to be; their provenance and authorship are known and verified" (p. 363). Authenticity is intimately connected with modernity where increasing personal autonomy and social mobility rendered necessary the "labor of self-definition" (Hartman, 1973: see also Trilling, 1972). Digital sociality creates new venues and visualities for the "labour of self-definition": it also reinvigorates deeper, older anxieties about photography and authenticity. $\subseteq$

5. See https://www.urbandictionary.com/define.php?term=Plandid $\leftrightarrows$

6. https://www.getolympus.com/us/en/learn_center/plandid-instagram-trend $€$ 7. "Watermarking" an invented term within peer group discourses does not appear to have widespread use. $\doteq$

8. We have protected the identities of all participants with pseudonyms.

9. Recruiting methods used posters and social media calls for participation but also used snowballing, which resulted in par- ticipants knowing each other on occasion. $\triangleq$

\section{Citations}

1. Nietzsche, F. W. (2000). The birth of tragedy (Smith, D. , Trans.). Oxford University Press. $\subseteq$

2. Leclercq, C. (2016). Do you "LensIt"? A call for research on modified selfies. On Masters of Media blog $\subseteq$

3. Cambre, C., Lavrence, C. (2019). How else would you take a photo? \#SelfieAmbivalence. Cultural Sociology, 13(4), 503-524.

4. Diamond, A. (2017). The most indulgent Instagram trend of 2017. https://www.manrepeller.com/2017/07/celebrity-instagram-poses.html 5. Cheng, A. (2017). What is a plandid? Here's why celebrities are all over this Instagram trend. https://www.billboard.com/articles/news/lifestyle/7948968/instagram-posing=

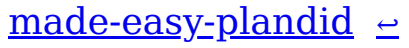


6. Hevesi, D. (2012, November 28). Bryce Bayer, inventor of a filter to make colour digital pictures, dies at 83. The New York

Times. https://www.nytimes.com/2012/11/29/business/bryce-bayer-inventor-of-a-filterto-make-color-digital-pictures-dies-at-83.html $\triangleq$

7. Britannica. (2019, May 16). Adobe photoshop. Encyclopedia

Britannica. www.britannica.com/technology/Adobe-Photoshop

8. Schipper, M. (2018). "A whole new way to see yourself (ie)": Exploring how face filters transform the practice of selfie creation [Unpublished Master's thesis, Utrecht University]. $\triangleq$

9. Evans, A., Riley, S. (2013). Immaculate consumption: Negotiating the sex symbol in postfeminist celebrity culture. Journal of Gender Studies, 22(3), 268-281. 10. Bakhshi, S., Shamma, D. A., Gilbert, E. (2014). Faces engage us: Photos with faces attract more likes and comments on Instagram [Conference session]. The SIGCHI Conference on Human Factors in Computing Systems, pp. 965974. http://comp.social.gatech.edu/papers/chi14.faces.bakhshi.pdf $\triangleq$ 11. Bakhshi, S., Shamma, D., Kennedy, L., Gilbert, E. (2015). Why we filter our photos and how it impacts engagement [Conference session]. Ninth International AAAI Conference on Web and Social Media. http://comp.social.gatech.edu/papers/icwsm15.why.bakhshi.pdf $\subseteq$ 12. Barnard, S. R. (2016). Spectacles of self(ie) empowerment? Networked individualism and the logic of the (post)feminist selfie. In Robinson, L., Cotton, S. R., Schulz, J. (Eds.), Communication and information technologies annual (Studies in media and communications, 11, pp. 63-88). Emerald. $\subseteq$

13. Rettberg, J. W. (2014). Seeing ourselves through technology: How we use selfies, blogs and wearable devices to see and shape ourselves. Palgrave Macmillan. 14. Elias, A. S., Gill, R. (2018). Beauty surveillance: The digital self-monitoring cultures of neoliberalism. European Journal of Cultural Studies, 21(1), 59-77. 15. Dhir, A., Pallesen, S., Torsheim, T., Andreassen, C. S. (2016). Do age and gender differences exist in selfie-related behaviours? Computers in Human Behavior, 63(C), 549-555. 
16. Kedzior, R., Allen, D. E. (2016). From liberation to control: Understanding the selfie experience. European Journal of Marketing, 50(9/10), 1893-1902. 17. Vivienne, S. (2017). "I will not hate myself because you can't accept me": Problematizing empowerment and gender diverse selfies. Popular Communication, 15(2), 126-140.

18. Gill, R. (2007). Postfeminist media culture: Elements of a sensibility. European Journal of Cultural Studies, 10(2), 147-166.

19. Gill, R. (2016). Post-feminism? New feminist visibilities in postfeminist times. Feminist Media Studies, 16(4), 610-630.

20. Gill, R. (2017). The affective, cultural and psychic life of postfeminism: A postfeminist sensibility 10 years on. European Journal of Cultural Studies, 20(6), 606626. $\triangleq$

21. Gill, R., Scharff, C. (Eds.). (2011). New femininities: Postfeminism, neoliberalism and subjectivity. Palgrave Macmillan. $\triangleq$

22. Ringrose, J., Coleman, R. (2013). Looking and desiring machines: A feminist Deleuzian mapping of bodies and affects. In Coleman, R., Ringrose, J. (Eds.), Deleuze and research methodologies (pp. 124-144). Edinburgh University Press. $ヒ$ 23. Riley, S., Evans, A., Mackiewicz, A. (2016). It's just between girls: Negotiating the postfeminist gaze in women's "looking talk." Feminism \& Psychology, 26(1), 94113.

24. Senft, T. (2015). The skin of the selfie. In Bieber, A. (Ed.), Ego update: A history of the selfie (pp. 134-160). NRW Forum. 25. Sartre, J. -P. (1957). Being and nothingness (Barnes, H. , Trans.). Philosophical Lib. (Original work published 1943) 26. Lacan, J. (1978). Four fundamental concepts in psychoanalysis (Miller, JacquesAlain , Ed. and Sheridan, A. , Trans.). Norton. $\doteq$ 27. Butler, J. (1990). Gender trouble: Feminism and the subversion of identity. Routledge. $\leftrightarrows$ 28. Foucault, M. (1977). Panopticism. In Foucault, M. (Ed.), Discipline and punish: The birth of the prison (pp. 195-228). Vintage Books. $\subseteq$ 
29. Mulvey, L . (1989). Visual pleasure and the narrative cinema. In Mulvey, L. (Ed.), Visual and other pleasures: Theories of representation and difference (pp. 1427). Indiana University Press. $\subseteq$ 30. hooks, b. (1992). The oppositional gaze: Black female spectators. In Jones, A. (Ed.), The feminism and visual culture reader (pp. 94-10). Routledge. $\subseteq$ 31. Kaplan, E. A. (1997). Looking for the other: Feminism, film and the imperial gaze. Routledge $ヒ$ 32. Said, E. (1978). Orientalism. Vintage Books. $\leftrightarrows$

33. Graybill, L. (2019). The forensic eye and the public mind. Cultural History, 81, 94-119.

34. Sekula, A. (1986). The body and the archive. October, 39, 3-64. 35. Ringrose, J., Renold, E. (2010). Normative cruelties and gender deviants: The performative effects of bully discourses for girls and boys in school. British Educational Research Journal, 36(4), 573-596. $ヒ$ 36. Jurgenson, N., Rey, P. J. (2010, April). Marcuse, the web, and the new means of ambient production[Paper presentation]. The VII Annual Social Theory Forum on Critical Social Theory: Freud \& Lacan for 21st Century, Boston, MA. 37. Rey, P. J. (2012). Alienation, exploitation and social media. American Behavioral Scientist, 56(4), 399-420. $匚$ 38. Deleuze, G., Guattari, F. (1987). A thousand plateaus (Massumi, B. , Trans.). University of Minnesota Press. $\subseteq$ 39. Peirce, C. S. (1985). Logic as semiotic: The theory of signs. In Innis, R. E. (Ed.), Semiotics: An anthology (pp. 4-23). Indiana University Press. $ヒ$ 40. Shields, R. (2004). Visualicity: On urban visibility and invisibility. Visual Culture in Britain, 5(1), 23-36.

41. Cambre, C., Lavrence, C. (2018). Encounters that open up problems: A critical path for post-structuralist inquiry. In Michalski, J. (Ed.), Sociological theory, methods, and perspectives (pp. 185-206). Nova Science Publishers. $\uplus$ 
42. Harper, D. (2002). Talking about pictures: A case for photo elicitation. Visual Studies, 17(1), 13-26.

43. Becker, H. (1974). Photography and sociology. Studies in the Anthropology of Visual Communication, 1, 3-26. $\doteq$

44. Pauwels, L . (2011). An integrated conceptual framework for visual social research. In Margolis, E., Pauwels, L. (Eds.), The SAGE handbook of visual research methods (pp. 3-23). SAGE.

45. Pauwels, L. (2015). Participatory' visual research revisited: A criticalconstructive assessment of epistemological, methodological and social activist tenets. Ethnography, 16(1), 95-117.

46. Ringrose, J., Renold, E. (2014). “F**k rape!”: Exploring affective intensities in a feminist research assemblage. Qualitative Inquiry, 20(6), 772-780. 47. Bal, M. (2002). Travelling concepts in the humanities: A rough guide. University of Toronto Press. $\doteq$

48. Gilbert, K. E. (2017). Policing evaluation: "Focus group interviews as an embodied speech event." Discourse \& Communication, 11(4), 341-361. 49. Natali, L. (2019). Visually exploring social perceptions of environmental harm in global urban contexts. Current Sociology, 67(5), 650-668. 50. Forsey, C. (2019, October 15). What's a Finsta? We explain this confusing trend. https://blog.hubspot.com/marketing/finsta $\subseteq$ 51. Bridges, T., Pascoe, C. J. (2014). Hybrid masculinities: New directions in the sociology of men and masculinities. Sociology Compass, 8(3), 246-258. 52. Hess, A. (2015). The selfie assemblage. International Journal of Communication, 9, 1629-1646. $\uplus$

53. Peterson, D. (n.d.). How to take a great selfie. http://www.digital-photosecrets.com/tip/3743/take-great-selfie/ $ヒ$ 54. Bourdieu, P. (1984). Distinction: A social critique of the judgement of taste. Harvard University Press. $\doteq$ 
55. Winch, A. (2012, December 4). The girlfriend gaze.

Eurozine. https://www.eurozine.com/the-girlfriend-gaze/ $\triangleq$

56. Senft, T. (2018). Hating Habermas: On exhibitionism, shame, \& life on the actually existing internet. http://eitherand.org/exhibitionism/hating-habermasexhibitionism-shame-life-actually-l $\triangleq$

57. Grogan, S., Rothery, L., Cole, J., Hall, M. (2018). Posting selfies and body image in young adult women: The selfie paradox. The Journal of Social Media in Society, $1(1), 15-36$.

58. Schroeder, J. (2013). Snapshot aesthetics and the strategic imagination. Invisible Culture. https://ivc.lib.rochester.edu/snapshot-aesthetics-and-the-strategicimagination/ $\triangleq$ 